\title{
Huntingtin-associated protein 1: A potential biomarker of breast cancer
}

\author{
LIWEI ZHU ${ }^{1,2}$, XUE SONG ${ }^{1,2}$, JINHAI TANG $^{1}$, JIANZHONG WU $^{2}$, RONG MA ${ }^{2}$, \\ HAIXIA CAO ${ }^{2}$, MINGHUA JI ${ }^{3}$, CHANGWEN JING ${ }^{2}$ and ZHUO WANG $^{2}$ \\ ${ }^{1}$ Department of General Surgery, the Affiliated Jiangsu Cancer Hospital; ${ }^{2}$ Research Center of Clinical Oncology, \\ the Affiliated Jiangsu Cancer Hospital; ${ }^{3}$ Department of Radiotherapy, the Affiliated Jiangsu \\ Cancer Hospital, Nanjing Medical University, Nanjing, Jiangsu 210000, P.R. China
}

Received November 8, 2012; Accepted December 21, 2012

DOI: $10.3892 /$ or.2013.2303

\begin{abstract}
It is reported that patients with Huntington's disease (HD) have a low incidence of cancer. In this study, we investigated the expression of huntingtin-associated protein 1 (HAP1), the ligand of HD's production, in breast tumor and normal tissues. We found that HAPl expression was significantly lower in tumor compared to normal tissues. We then transfected the HAPl gene into the breast cancer lines MCF-7 and MDA-MB-231, and results showed that the overexpression of $H A P 1$ reduced the growth of the two cell lines. In addition, we observed that $H A P 1$ also reduced invasion and migration, and upregulated apoptosis in MCF-7 cells; however, these changes were not observed in MDA-MB-231 cells. We also demonstrated that the expression of EGFR and apoptosis-related genes might be involved in cell proliferation and apoptosis. In conclusion, overexpression of $H A P 1$ reduced in vitro cell growth in breast cancer cell lines, suppressed the migration and invasion, and promoted the apoptosis of certain cell lines. Therefore, HAPl is a potential molecular target for the diagnosis and treatment of breast cancer.
\end{abstract}

\section{Introduction}

Breast cancer is the most prevalent tumor in women, and its incidence accounts for $7-10 \%$ of all malignant tumors. Huntington's disease (HD) is an autosomal dominant hereditary neurodegenerative disorder characterized by late onset, progressive psychiatric disruption, cognitive deficits and loss of motor coordination (1). According to a previous study, the overall incidence of cancer is significantly lower among patients with HD, and this seems to be related to intrinsic biologic factors (2). HD is caused by a CAG triplet repeat expansion in exon 1 of the huntingtin (Htt) gene, encoding an abnormal

Correspondence to: Professor Jinhai Tang, Department of General Surgery, the Affiliated Jiangsu Cancer Hospital, Nanjing Medical University, 42 Bai Zi Ting Road, Nanjing, Jiangsu 210000, P.R. China

E-mail: tangjh_dr@126.com

Key words: huntingtin-associated protein 1, invasion, migration, apoptosis, breast cancer expanded polyglutamine (polyQ) tract that confers toxicity to the mutant Htt protein. When the mutant Htt gradually accumulates in the cell, it can affect the function of several proteins and the conduction of some signaling pathways. Huntingtininteracting protein 1 (HIP1) and huntingtin-associated protein 1 (HAP1) are two known ligands of Htt $(3,4)$. Research on the relevance between HIP1 and cancer showed that HIP1 had a high expression level in breast, colon, prostate and other types of cancer $(5,6)$. However, there are currently no studies regarding the association between HAP1 and cancer. In this study, we first examined the level of HAP1 in human breast tumor and normal breast tissues, and investigated the roles and possible mechanisms of HAP1 in human breast cancer cells. In addition, we selected ER positive MCF-7 and triple negative MDA-MB-231 cells, representing two main types of breast cancer, for research.

\section{Materials and methods}

Tumor samples. A total of 43 breast carcinoma specimens with non-neoplastic adjacent tissues from patients with primary breast tumor were collected during the surgical procedures at the Department of General Surgery, Jiangsu Cancer Hospital, Nanjing Medical University (Nanjing, China). The sample collection was carried out in accordance with the National Regulation of Clinical Sampling in China.

Cell culture. The human breast cancer cell lines MCF-7 and MDA-MB-231 were maintained in DMEM with high glucose (Gibco, Grand Island, NY, USA) and 10\% FBS (Gibco).

Viral production and infection of target cells. Retrovirus was generated by cotransfection of pBabe-puro empty vector (as control) or pBabe-puro-Hapl plasmid along with pVSP-G (envelope) and pVSV-GP (packaging) plasmids in 293FT cells, growing at $85-90 \%$ confluency in $10-\mathrm{cm}$ petri dishes. After $48 \mathrm{~h}$, the culture medium containing the viral particles was harvested. Target cells were infected with virus containing medium in the presence of $10 \mu \mathrm{g} / \mathrm{ml}$ polybrene (Sigma, St. Louis, MO, USA). Then, changing back to fresh medium, cells were selected with $2 \mu \mathrm{g} / \mathrm{ml}$ puromycin (Fischer, USA) for 7-10 days, re-cultured in a larger dish containing puromycin 
Table I. Primers used for Q-PCR amplification.

\begin{tabular}{lll}
\hline Gene & \multicolumn{1}{c}{ Forward } & \multicolumn{1}{c}{ Reverse } \\
\hline HAP1 & 5'-ATGCGCCCGAAGAGGTTGG-3' & 5'-CTGCAGATCGTCGTGCCGATGA-3' \\
Caspase-3 & 5'-GATGGAAGCGAATCAATGGACT-3' & 5'-CTGTACCAGACCGAGATGTCA-3' \\
Caspase-8 & 5'-TCATGGACCACAGTAACATGGA-3' & 5'-AGTGAACTGAGATGTCAGCTCAT-3' \\
Caspase-9 & 5'-CACTCCCCTGAAGACGAGTC-3' & 5'-GTGGGCAAACTAGATATGGCG-3' \\
Bcl-2 & 5'-GGTGGGGTCATGTGTGTGG-3' & 5'-CGGTTCAGGTACTCAGTCATCC-3' \\
Bax & 5'-CCCGAGAGGTCTTTTTCCGAG-3' & 5'-CCAGCCCATGATGGTTCTGAT-3' \\
Survivin & 5'-TGCGGGAATCCAAAGGATAATTCA-3' & 5'-CTTCATCTTTGTCATACTTCATGGCT-3' \\
EGFR & 5'-GAAGGAGCTGCCCATGAGAA-3' & 5'-GACTATGTCCCGCCACTGGAT-3' \\
$\beta$-actin & 5'-TTCTACAATGAGCTGCGTGTG-3' & 5'-CAGCCTGGATAGCAACGTACA-3' \\
\hline
\end{tabular}

HAP1, huntingtin-associated protein 1 .

and used for further assay. All plasmids and cells were kindly provided by Professor Jinrong Zhou, at Harvard University (Cambridge, MA, USA).

Cell viability assay. Cells (3,000/well) were plated in 96-well plates. Each group had five replicates. The growth was monitored $72 \mathrm{~h}$ later and cell viability was measured by CCK-8 (Dojindo, Kumamoto Prefecture, Kyushu, Japan).

Colony formation assay. Cells were seeded in six-well culture plates at 200 cells/well. After 10 days of incubation, the cells were stained with $0.1 \%$ crystal violet (Enox, Shanghai, China). The colonies with $>50$ cells were counted. The colony formation rate was acquired through colony numbers/total seeding cells.

Semiquantitative RT-PCR. Total RNA was extracted with the TRIzol Reagent (Invitrogen, Carlsbad, CA, USA) and was then reverse transcribed using PrimeScript RT Master mix (Takara, Kumamoto Prefecture, Kyushu, Japan). Real-time PCR was performed using an ABI 7300 Real-time PCR system (Applied Biosystems, Carlsbad, CA, USA) with the SYBR Premix Ex Taq (Takara). All samples were analyzed in triplicate and in optically clear 96-well plates (Corning Inc., Corning, NY, USA). The cycling parameters were: $95^{\circ} \mathrm{C}$ for $30 \mathrm{sec}$ followed by 40 cycles of $95^{\circ} \mathrm{C}$ for $5 \mathrm{sec}, 60^{\circ} \mathrm{C}$ for $31 \mathrm{sec}$ and $95^{\circ} \mathrm{C}$ for $15 \mathrm{sec}, 60^{\circ} \mathrm{C}$ for $60 \mathrm{sec}, 95^{\circ} \mathrm{C}$ for $15 \mathrm{sec}$. The human $\beta$-actin transcript was used as an internal reference to control for variations in the total mRNA quantity of each sample. Each RNA sample was analyzed in triplicate. Primer sequences are listed in Table I.

Western blot analysis. Cells were collected and lysed with lysis buffer (Beyotime, Jiangsu, China), and concentrated to obtain the proteins. The amount of total proteins was estimated by Onedrop OD-1000+ Spectrophotometer (Onedrop, Shanghai, China), and then proteins were mixed with SDS-PAGE buffer (Beyotime) and boiled for $5 \mathrm{~min}$. The proteins were loaded onto $10 \%$ SDS-PAGE gel and, following electrophoresis, the proteins were transferred onto a PVDF membrane (Bio-Rad, Hercules, CA, USA). Then, the membrane was blocked with nonfat-dried milk, incubated with primary and secondary antibody (Dako, Japan), and protein bands were visualized by ECL detection reagent (Millipore, Billerica, MA, USA).

In vitro migration and invasion assays. For the migration assays, the cells were detached and aliquots of $2 \times 10^{5}$ cells $/ \mathrm{ml}$ were plated onto the inserts of the $8-\mu \mathrm{m}$ pore-sized Transwell chambers (Corning Inc.). For the Transwell invasion assays, $1 \times 10^{6}$ cells were plated onto inserts containing a polycarbonate membrane with a thin layer of BD Matrigel Matrix (BD Biosciences, Franklin Lakes, NJ, USA). Both migration and invasion were assayed $24 \mathrm{~h}$ later by counting the cell numbers across the membrane.

Flow cytometric assay. For cell apoptosis, a total of $1 \times 10^{6}$ cells were transferred to a tube in which $5 \mu \mathrm{l}$ Annexin $\mathrm{V}$ and $1 \mu \mathrm{l}$ propidium iodide (PI) were added after being resuspended with $100 \mu \mathrm{l}$ binding buffer (Invitrogen). The cells were then allowed to incubate at room temperature for $15 \mathrm{~min}$ and were analyzed using flow cytometry. For cell cycle analysis, cells were collected, washed twice with PBS, supplemented with $1 \mathrm{ml} 75 \%$ ethanol, and kept at $-20^{\circ} \mathrm{C}$ overnight. The cells were then resuspended with PBS, supplemented with PI and RNase A, incubated for $30 \mathrm{~min}$ at $37^{\circ} \mathrm{C}$ and analyzed using flow cytometry.

Statistical analyses. Data are expressed as the means \pm SD, and statistical significance was assessed by Student's t-test and one-way ANOVA. $\mathrm{P}<0.05$ was considered to indicate a statistically significant difference.

\section{Results}

Expression of HAPl in breast tumor samples. HAPl expression was assessed by qRT-PCR in surgical specimens from patients with breast cancer. As shown in Fig. 1, HAPl expression was markedly reduced in most cancer tissues compared with normal breast tissues. Of 43 cases, $70 \%$ showed HAP1 expression levels in normal breast tissues $>3$-fold higher than those of matched tumors (Fig. 1).

Expression of HAP1 in transfected and control cells. The MCF-7 and MDA-MB-231 cells transfected with pBabe-puro-HAP1 


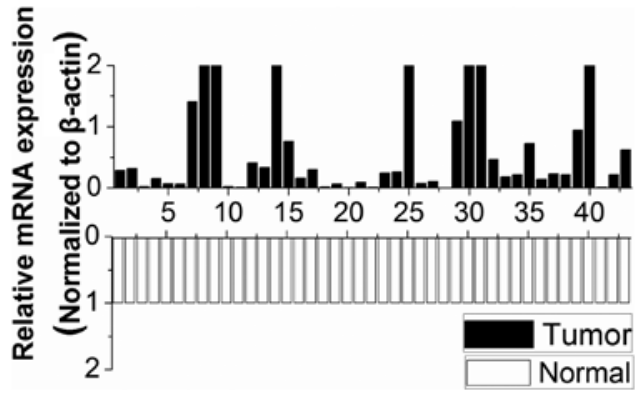

Figure 1. Huntingtin-associated protein $1(H A P 1)$ expression in breast tumor and matched normal breast tissues. HAP1 expression in 43 cases of breast tumor and matched normal breast tissues was evaluated. The HAP1 mRNA expression of the majority of the normal breast tissue $(70 \%)$ was $>3$-fold higher compared with matched tumors.

A

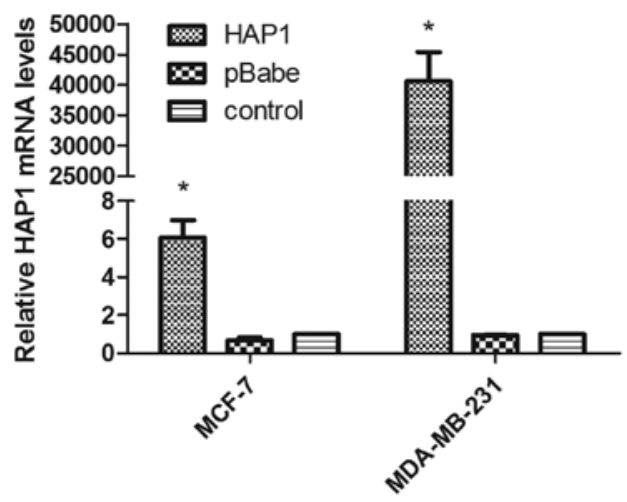

B

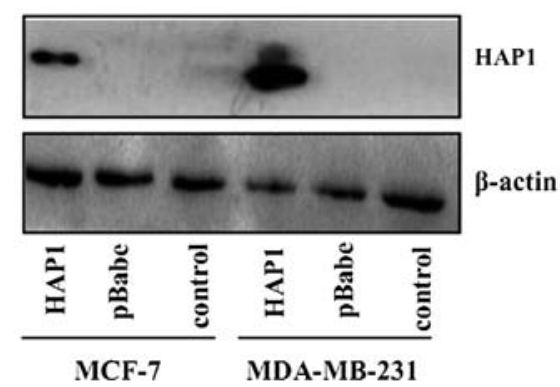

Figure 2. Huntingtin-associated protein 1 (HAP1) expression in transfected and control cells lines. (A) Relative mRNA levels of $H A P 1$ in all cell lines. (B) Western blot analysis of HAP1 levels in all cell lines. "P<0.05.

and pBabe-puro were labeled MCF-7/HAP1, MCF-7/pBabe and MDA-MB-231/HAP1, MDA-MB-231/pBabe, respectively. Western blotting and qRT-PCR results of different cells showed that MCF-7/HAP1 $(\mathrm{P}<0.006)$ and MDA-MB-231/HAP1 $(\mathrm{P}=0.001)$ had a significantly higher expression of HAP1 than their control groups. Moreover, there was no obvious difference between pBabe and negative control groups $(\mathrm{P}>0.05)$ (Fig. $2 \mathrm{~A}$ and $\mathrm{B})$.

Effects of HAP1 on breast cancer cell growth and expression of EGFR in cells. At $72 \mathrm{~h}$ after cells were plated in 96-well plates, upregulation of $H A P 1$ significantly increased the cell viability of both $H A P 1$ overexpression cells in MCF-7 [HAP1 mean, 76.0 \pm 6.8 vs. pBabe mean, 94.0 $\pm 6.6(\mathrm{P}=0.03)$ vs. control mean, $100 \pm 0.0(\mathrm{P}=0.004)]$ and MDA-MB-231 [HAP1 mean, 68.2 \pm 17.4 vs. pBabe mean, 99.8 $\pm 6.0(\mathrm{P}=0.041)$

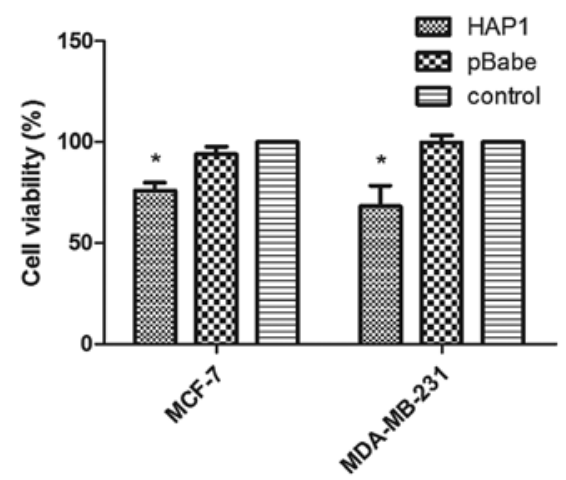

B

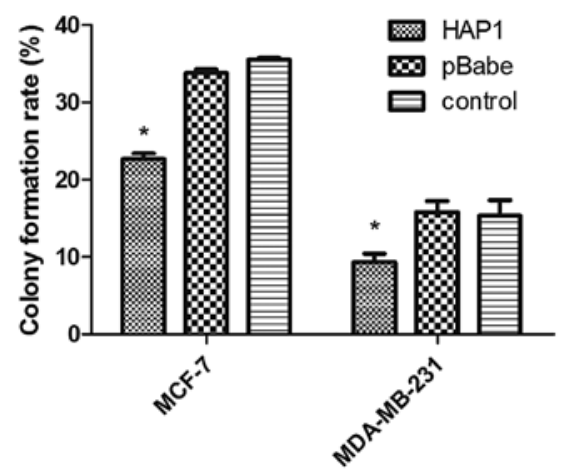

C

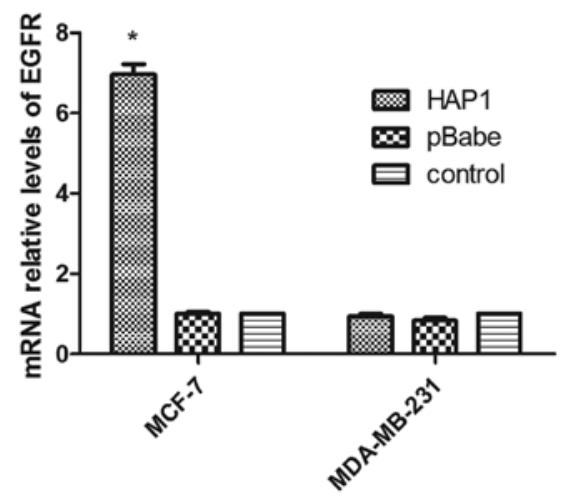

Figure 3. Growth and proliferation ability of cells and EGFR expression levels in cells. (A) Cell viability test of the two cell line groups. (B) Quantitative evaluation of colony formation in the two cell line groups, indicated as the ratio to control cells. (C) Significantly higher expression of EGFR in MCF-7/HAP1, but not in MDA-MB-231/HAP1. "P<0.05. HAP1, huntingtinassociated protein 1 .

vs. control mean, $100 \pm 0.0(\mathrm{P}=0.034)]$ compared with empty vector plasmid transfected cells and negative control cells (Fig. 3A). In addition, colony formation rates were significantly decreased in MCF-7 cells with HAP1 overexpression [HAP1 mean, $22.7 \pm 1.3 \%$ vs. pBabe mean, $33.8 \pm 0.8 \%(\mathrm{P}=0.000)$ vs. control mean, $35 \pm 0.5 \%(\mathrm{P}=0.000)$ ] and MDA-MB-231 [HAP1 mean, $9.3 \pm 1.9 \%$ vs. pBabe mean, $15.8 \pm 2.5 \%(\mathrm{P}=0.022)$ vs. control mean, $16 \pm 2.6 \%(\mathrm{P}=0.024)]$ when compared with those of pBabe and control cells (Fig. 3B). We also compared the expression of EGFR in three groups of the cell lines. We observed that in the MCF-7 cell lines, EGFR expression of MCF-7/HAP1 [HAP1 mean, $7.0 \pm 0.4$ vs. pBabe mean, $1.0 \pm 0.1$ $(\mathrm{P}=0.000)$ vs. control mean, $1.0 \pm 0.0(\mathrm{P}=0.000)]$ was $\sim 7$-fold higher than that of the other two groups. However,there was no difference in EGFR expression between the three groups in the MDA-MB-231 cell lines ( $\mathrm{F}=3.2 ; \mathrm{P}=0.1)$ (Fig. 3C). 
A

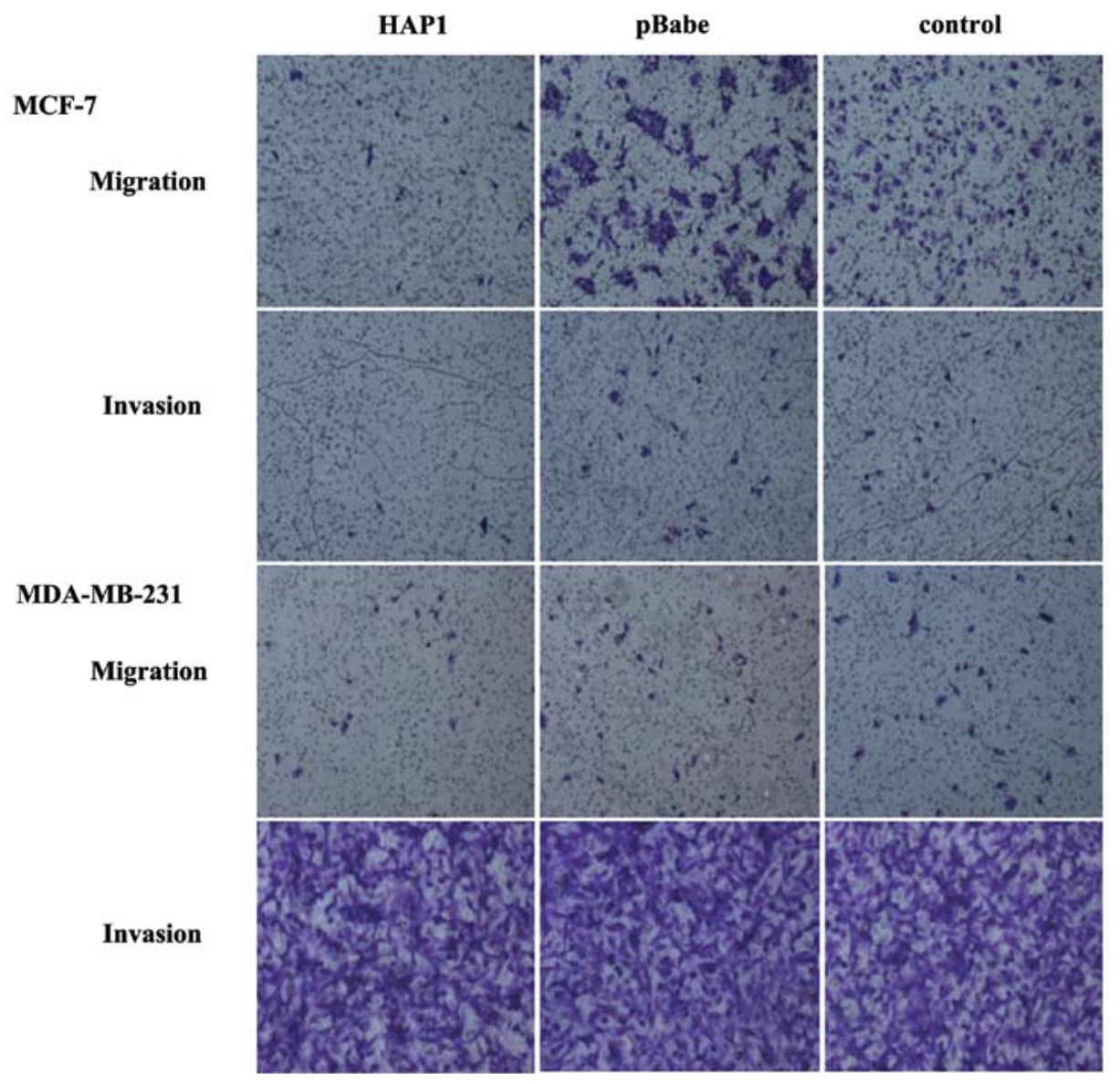

B

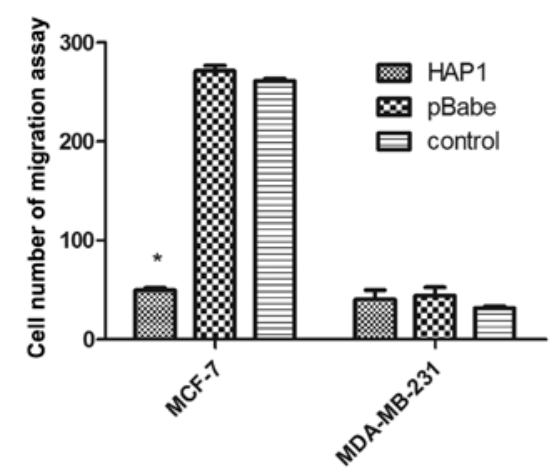

C

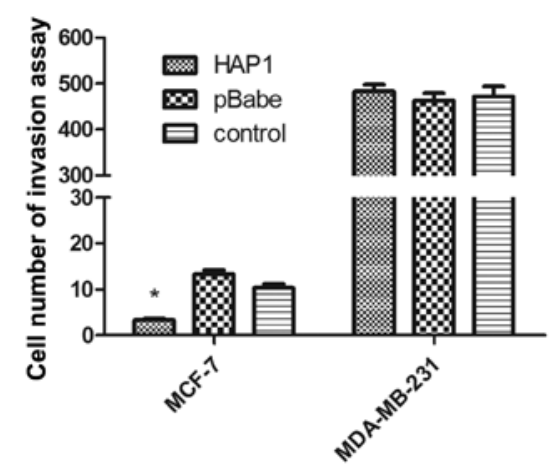

Figure 4. Effect of huntingtin-associated protein 1 (HAPI) overexpression on the migration and invasion ability of MCF-7 and MDA-MB-231 cells. (A) Images of representative crystal violet-stained membrane of migration and invasion, x200. In MCF-7 cells, migration and invasion in the HAP1 group were reduced compared with the other two groups. No changes were observed in MDA-MB-231 cells. (B) Quantitative evaluation of cell migration ability (chambers without Matrigel); the migrating cells were counted in six predetermined fields. (C) Quantitative evaluation of cell invasion ability (chambers with Matrigel); the invading cells were counted in six predetermined fields. ${ }^{*} \mathrm{P}<0.05$.

Overexpression of HAP1 impairs migration and invasion. We observed that MCF-7/HAP1 had fewer cells across the membrane than MCF-7/pBabe and MCF-7 in migration assays [HAP1 mean, 50.0 \pm 3.6 vs. pBabe mean, $271 \pm 9.5(\mathrm{P}=0.000)$ vs. control mean, 261 $\pm 4.6(\mathrm{P}=0.000)]$ (Fig. $4 \mathrm{~A}$ and $\mathrm{B})$. In addition, compared to the number of the other two cell groups, the number of MCF-7/HAP1 cells that invaded the Matrigelcoated filter was significantly lower [HAP1 mean, $3.3 \pm 0.6$ vs. pBabe mean, $13.3 \pm 1.5(\mathrm{P}=0.000)$ vs. control mean, $10.3 \pm 1.5$ $(\mathrm{P}=0.000)]$ (Fig. 4A and C). Thus, the overexpression of HAPI in MCF-7 can curb tumor cell migration and invasion. However, in MDA-MB-231 cells, there was no significant difference in the number of cells across the membrane in both cell migration $(\mathrm{F}=0.8 ; \mathrm{P}=0.5)$ and invasion $(\mathrm{F}=0.3 ; \mathrm{P}=0.7)(\mathrm{Fig} .4 \mathrm{~A}-\mathrm{C})$.

Effects of HAP1 on cell cycle, apoptosis and expression of relative genes. According to cell cycle distribution analysis by flow cytometry, HAP1 increased the percentage of cells in the $\mathrm{G}_{2} \mathrm{M}$ phase. In MCF-7 cells, the percentage in the $\mathrm{G}_{2} \mathrm{M}$ phase of the HAP1 group was $30.84 \%$, higher than the $13.17 \%$ and the $10.61 \%$ in the pBabe and control groups. In MDA-MB-231 cells, the percentage in the $\mathrm{G}_{2} \mathrm{M}$ phase of the HAP1 group was $14.84 \%$, also higher than the other two groups (Table II). The results indicated that HAP1 induced $\mathrm{G}_{2} \mathrm{M}$ arrest in both 
Table II. Cell cycle distribution in MCF-7 and MDA-MB-231 cells.

\begin{tabular}{|c|c|c|c|c|c|c|}
\hline & \multicolumn{3}{|c|}{$\mathrm{MCF}-7$} & \multicolumn{3}{|c|}{ MDA-MB-231 } \\
\hline & HAP1 & pBabe & Control & HAP1 & pBabe & Control \\
\hline $\mathrm{G}_{0} \mathrm{G}_{1}(\%)$ & $36.53 \pm 0.96$ & $46.04 \pm 2.22$ & $58.11 \pm 2.18$ & $56.69 \pm 0.83$ & $56.79 \pm 1.08$ & $55.63 \pm 1.02$ \\
\hline $\mathrm{S}(\%)$ & $32.63 \pm 0.34$ & $40.78 \pm 0.41$ & $31.27 \pm 0.66$ & $28.46 \pm 0.22$ & $31.45 \pm 0.22$ & $33.17 \pm 0.17$ \\
\hline $\mathrm{G}_{2} \mathrm{M}(\%)$ & $30.84 \pm 1.09^{\mathrm{a}}$ & $13.17 \pm 1.83$ & $10.61 \pm 1.53$ & $14.84 \pm 0.90^{\mathrm{a}}$ & $11.76 \pm 0.93$ & $11.19 \pm 0.94$ \\
\hline
\end{tabular}

${ }^{\mathrm{a}} \mathrm{P}<0.05$.

A
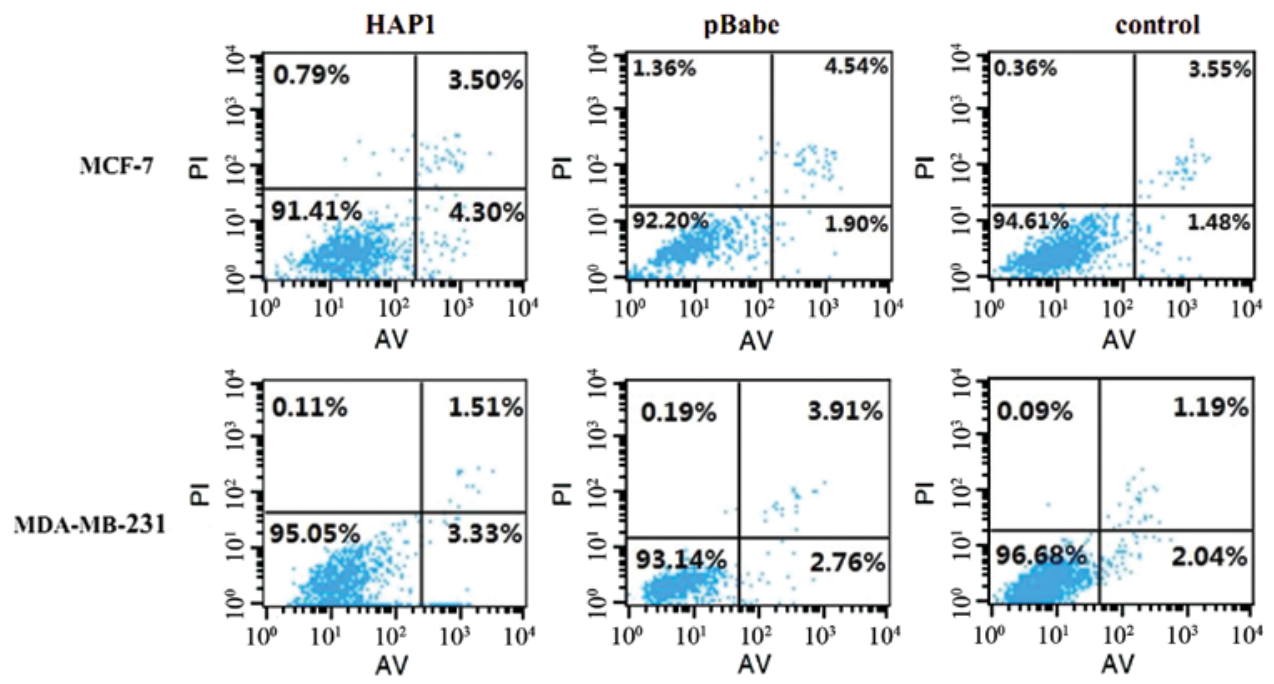

B

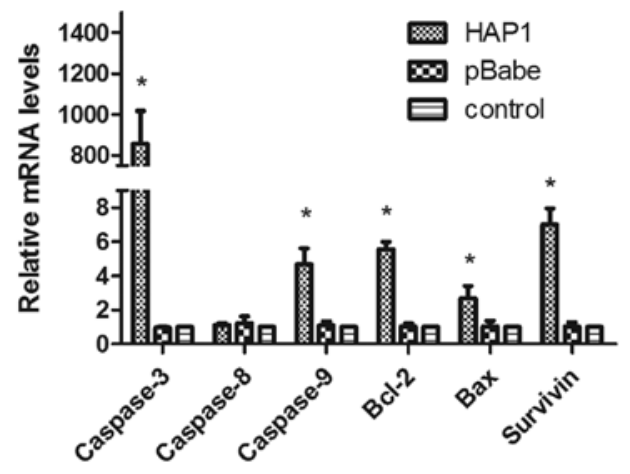

MCF-7

D

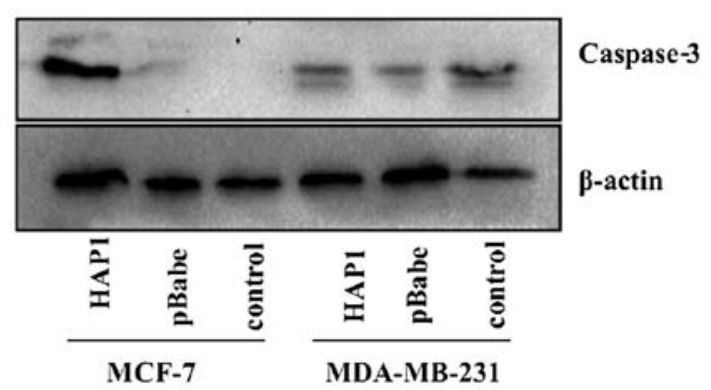

C

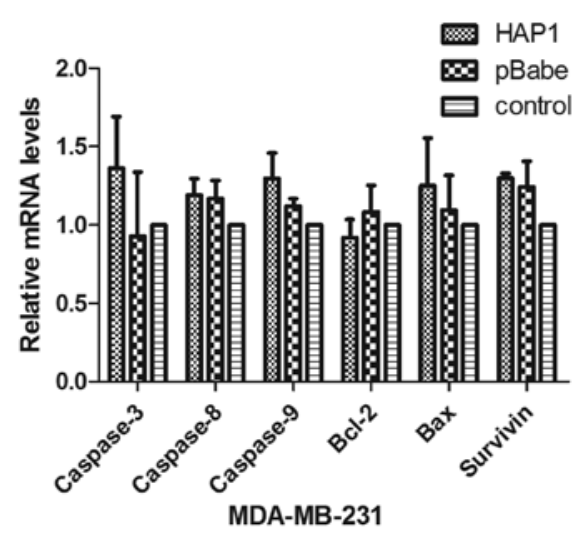

Figure 5. Overexpression of huntingtin-associated protein 1 (HAPl) induces cell cycle arrest in both cells and apoptosis in MCF-7, but not in MDA-MB231 , and regulates the signaling related to apoptosis and survival. (A) By flow cytometric assay, overexpression of HAP1 in MCF-7 cells induced significant apoptosis compared with pBabe and control groups. However, we did not find any significant difference in apoptosis in the three groups of the MDAMB-231 cells. Upper left, dead cells; upper right, dead/late apoptotic cells; lower left, normal cells; lower right, early apoptotic cells. (B) Apoptosis and survival relative genes were more highly expressed in MCF-7/HAP1 than in MCF-7/pBabe and control groups. (C) There was no significant difference in the apoptosis and survival relative gene mRNA expression between the three groups in MDA-MB-231 cells. (D) Expression of caspase-3 was examined by immunoblotting, which was in accordance with the RT-PCR results. ${ }^{*} \mathrm{P}<0.05$.

expression of genes, including caspase-3, -8, -9, Bcl-2, Bax and survivin, involved in cell apoptosis and survival, by RT-PCR (Fig. 5B and C). The results showed that the expression of caspase-3, -9, Bcl-2, Bax and survivin in MCF-7/HAP1 was cell lines. Furthermore, $H A P 1$ overexpression significantly induced apoptosis of MCF-7 cells compared with pBabe and control groups, and there was also no significant difference in MDA-MB-231 cells (Fig. 5A). Therefore, we detected the 
significantly higher than in the other two groups. Moreover, the expression of caspase-3 was $>800$ times higher in MCF-7/ HAP1 compared with the other two groups [HAP1 mean, $857.7 \pm 278.9$ vs. $p B a b e$ mean, $1.0 \pm 0.1(\mathrm{P}=0.000)$ vs. control mean, $1.0 \pm 0.0(\mathrm{P}=0.000)]$, and it was confirmed by western blotting (Fig. 5D).

\section{Discussion}

HAP1 is a type of cytoplasmic protein distributed mainly in the microtubules and membranous organelles, including the mitochondria, endoplasmic reticulum, tubulovesicles, endosomal and lysosomal organelles, and synaptic vesicles $(7,8)$. HAP1 affects the synthesis of certain proteins and the conduction of signals (9-11). As a ligand of the production of HD, it was initially studied in the nervous system, whereas later research showed that it was involved in the other systems.

Herein, we detected the expression of HAPI in breast tumor and normal breast tissues. In contrast to normal breast tissues from breast cancer patients, we observed that HAPI expression was reduced in the majority of breast tumor samples. To the best of our knowledge, this is the first published study to show that $H A P I$ is downregulated in breast tumor tissues and, based on these findings, it is suggested that HAPl plays a role in the pathogenesis of breast cancer.

HAP1 is involved in numerous cellular functions, including cell proliferation and apoptosis $(12,13)$. In this study, upregulation of HAPl resulted in a significant decrease in the cell growth and colony formation rate of both breast cancer cells. Furthermore, cell cycle results showed that the percentage of $\mathrm{G}_{2} \mathrm{M}$ phase increased in both MCF-7 and MDA-MB-231 cells, indicating that cell growth could be arrested at the $\mathrm{G}_{2} \mathrm{M}$ phase by HAP1. Moreover, apoptosis may be involved in the inhibition of cell growth. It has been reported that apoptosis plays an important role during the malignant transformation of normal cells. The above studies, along with ours, indicate that loss or downregulation of HAPI may disrupt the balance between proliferation and apoptosis and may represent a key pathogenic step in the development of breast cancer. The cell apoptosis assay confirmed that HAP1 induced cell apoptosis and increased expression of relative genes in MCF-7 cells, particularly caspase-3. Caspase- 3 is located at the downstream of the apoptosis signaling pathway, and plays a key role in cell apoptosis $(14,15)$. Collectively, HAP1-induced apoptosis in MCF-7 cell lines may be mainly mediated by the caspasedependent pathway; in the MDA-MB-231 cells, however, the three groups showed no difference in the expression of those genes.

Previous studies showed that inhibition of HAPl expression decreased EGFR signaling and cell viability, whereas overexpression of HAPl enhanced this signaling activity (12). EGFR is expressed in various types of tissue, including epithelial, mesenchymal and of neuronal origin, and plays a major role in normal cellular processes such as proliferation, differentiation and development. EGFR is also highly expressed in a number of solid tumors and its expression correlates with tumor progression, resistance to chemotherapy and a poor prognosis $(16,17)$. Our detection of EGFR showed that EGFR expression was higher in MCF-7/HAP1, which negatively correlated with the results of cell proliferation. Therefore,

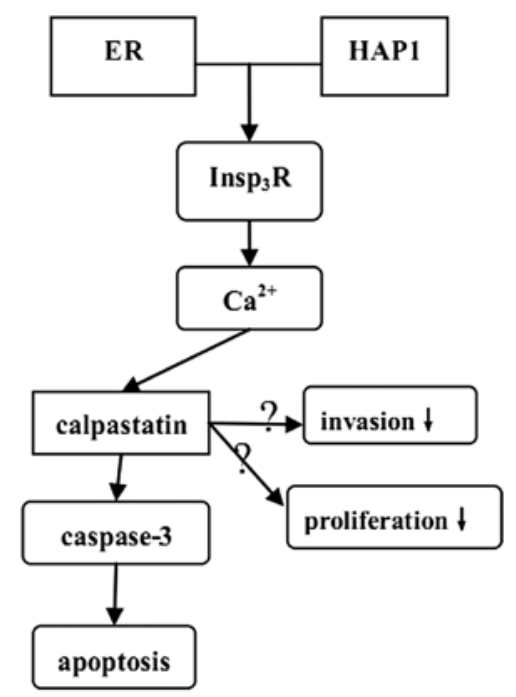

Figure 6. Model of our hypothesis showing how huntingtin-associated protein 1 (HAP1) affects MCF-7 but not MDA-MB-231 cells.

we considered that EGFR might play a role in MCF/HAP1, although not a major one; on the contrary, the effect of EGFR was counteracted by others.

Although it is unclear why the effect of HAP1 is significant in MCF-7 cells and not in MDA-MB-231 cells, we propose a hypothesis (Fig. 6). It was reported that more than $90 \%$ sporadically lurking HAP1-immunoreactive (HAP1-ir) cells expressed nuclear estrogen receptor (ER) (18). Therefore, we hypothesized that ER-positive MCF-7 cells gathered more HAP1 than triple negative MDA-MB-231 cells and interacted with them.

In various cells, the $\mathrm{Ca}^{2+}$ signaling process is mediated by the endoplasmic-reticulum-membrane-associated 1,4,5-trisphosphate $\left(\operatorname{Insp}_{3}\right)$ receptor $\left(\operatorname{Insp}_{3} R\right)$, which has a critical role in the control of cellular and physiological processes as diverse as cell division, cell proliferation and apoptosis $(19,21)$. Tang et al found that $\mathrm{Insp}_{3} \mathrm{R}$ could bind with HAP1A in vitro and in vivo, suggesting that HAP1 may impact the function of $\operatorname{Insp}_{3} R(20)$. When $\operatorname{Insp}_{3} R$ is activated by HAP1, the $\mathrm{Ca}^{2+}$ release is increased, subsequently inducing the activation of calpains.

The calpains are a family of neutral cysteine proteases that require calcium for their catalytic activity. The cellular activity of calpain is, in part, regulated by their endogenous inhibitor calpastatin $(22,23)$. The calpains function in the controlled proteolysis of a large number of specific substrates involved in various cellular processes such as migration, cell signalling and apoptosis. In addition, previous studies showed that calpain cleaved caspase-9 and therefore blocked the caspase-3 activation (24). Furthermore, Storr et al suggested that ER-positive breast cancer had a significantly high calpastatin expression compared with ER-negative breast cancer, and the expression of calpastatin in non-triple negative breast cancer was also significantly higher than in triple-negative breast cancer (22). The expression of calpain 1, however, was the opposite of calpastatin. Based on these data, we deduced that in ER-positive or (and) non-triple negative breast cancers, more apoptosis was induced than in ER-negative or (and) triple-negative breast cancer. 
Collectively, ER, expressed in MCF-7, induced HAP1 assembly, then sequentially activated $\operatorname{Insp}_{3} R$, calpastatin and caspase-3, finally increasing apoptosis. Moreover, the procedure may also impact invasion. This pathway does not exist in MDA-MB-231. However, further research is required to verify the hypothesis and to clarify the mechanism.

In summary, our findings demonstrated for the first time that HAPl downregulation occurs in breast tumor, and that HAP1 suppresses breast cancer cell growth. However, there were also some different effects of $H A P l$ between ER-positive MCF-7 and triple negative MDA-MB-231 cells, which we hypothesize may reflect the different types of breast cancer in the clinic. The findings of this study may provide a new therapeutic target for the treatment of breast cancer patients.

\section{Acknowledgements}

We thank Professor Jinrong Zhou of Harvard University and Dr Shuchun Li of Nanjing University of Technology for their technical assistance and helpful discussion.

\section{References}

1. Schulte J and Littleton JT: The biological function of the Huntingtin protein and its relevance to Huntington's Disease pathology. Curr Trends Neurol 5: 65-78, 2011.

2. Sorensen SA, Fenger K and Olsen JH: Significantly lower incidence of cancer among patients with Huntington disease: an apoptotic effect of an expanded polyglutamine tract? Cancer 86: 1342-1346, 1999.

3. Kalchman MA, Koide HB, McCutcheon K, et al: HIP1, a human homologue of $S$. cerevisiae Sla2p, interacts with membraneassociated huntingtin in the brain. Nat Genet 16: 44-53, 1997.

4. Li XJ, Li SH, Sharp AH, et al: A huntingtin-associated protein enriched in brain with implications for pathology. Nature 378: 398-402, 1995.

5. Rao DS, Hyun TS, Kumar PD, et al: Huntingtin-interacting protein 1 is overexpressed in prostate and colon cancer and is critical for cellular survival. J Clin Invest 110: 351-360, 2002.

6. Rao DS, Bradley SV, Kumar PD, et al: Altered receptor trafficking in Huntingtin Interacting Protein 1-transformed cells. Cancer Cell 3: 471-482, 2003.

7. Marcora E and Kennedy MB: The Huntington's disease mutation impairs Huntingtin's role in the transport of NF-kappaB from the synapse to the nucleus. Hum Mol Genet 19: 4373-4384, 2010.

8. Gutekunst CA, Li SH, Yi H, Ferrante RJ, Li XJ and Hersch SM: The cellular and subcellular localization of huntingtin-associated protein 1 (HAP1): comparison with huntingtin in rat and human. J Neurosci 18: 7674-7686, 1998.
9. Cape A, Chen X, Wang CE, et al: Loss of huntingtin-associated protein 1 impairs insulin secretion from pancreatic $\beta$-cells. Cell Mol Life Sci 69: 1305-1317, 2012.

10. Yang GZ, Yang M, Lim Y, et al: Huntingtin associated protein 1 regulates trafficking of the amyloid precursor protein and modulates amyloid beta levels in neurons. J Neurochem 122: 1010-1022, 2012

11. Yuen EY, Wei J, Zhong P and Yan Z: Disrupted GABAAR trafficking and synaptic inhibition in a mouse model of Huntington's disease. Neurobiol Dis 46: 497-502, 2012.

12. Li SH, Yu ZX, Li CL, et al: Lack of huntingtin-associated protein-1 causes neuronal death resembling hypothalamic degeneration in Huntington's disease. J Neurosci 23: 6956-6964, 2003.

13. Takeshita Y, Fujinaga R, Zhao C, Yanai A and Shinoda K Huntingtin-associated protein 1 (HAP1) interacts with androgen receptor (AR) and suppresses SBMA-mutant-AR-induced apoptosis. Hum Mol Genet 15: 2298-2312, 2006.

14. Galluzzi L, Kepp O and Kroemer G: Caspase-3 and prostaglandins signal for tumor regrowth in cancer therapy. Oncogene 31: 2805-2808, 2012.

15. Huang Q, Li F, Liu X, et al: Caspase 3-mediated stimulation of tumor cell repopulation during cancer radiotherapy. Nat Med 17: 860-866, 2011.

16. Li J, Pandey V, Kessler T, Lehrach H and Wierling C: Modeling of miRNA and drug action in the EGFR signaling pathway. PLoS One 7: e30140, 2012

17. Yano S, Kondo K, Yamaguchi M, et al: Distribution and function of EGFR in human tissue and the effect of EGFR tyrosine kinase inhibition. Anticancer Res 23: 3639-3650, 2003.

18. Islam MN, Fujinaga R, Yanai A, et al: Characterization of the 'sporadically lurking HAP1-immunoreactive (SLH) cells' in the hippocampus, with special reference to the expression of steroid receptors, GABA, and progenitor cell markers. Neuroscience 210: 67-81, 2012

19. Bosanac I, Alattia JR, Mal TK, et al: Structure of the inositol 1,4,5-trisphosphate receptor binding core in complex with its ligand. Nature 420: 696-700, 2002.

20. Tang TS, Tu H, Chan EY, et al: Huntingtin and huntingtin associated protein 1 influence neuronal calcium signaling mediated by inositol- $(1,4,5)$ triphosphate receptor type 1 . Neuron 39 : 227-239, 2003.

21. Kopil CM, Siebert AP, Kevin FJ and Neumar RW: Calpaincleaved type 1 inositol 1,4,5-trisphosphate receptor impairs $\mathrm{ER} \mathrm{Ca}(2+)$ buffering and causes neurodegeneration in primary cortical neurons. J Neurochem 123: 147-158, 2012.

22. Storr SJ, Lee KW, Woolston CM, et al: Calpain system protein expression in basal-like and triple-negative invasive breast cancer. Ann Oncol 23: 2289-2296, 2012.

23. Mataga MA, Rosenthal S, Heerboth S, et al: Anti-breast cancer effects of histone deacetylase inhibitors and calpain inhibitor. Anticancer Res 32: 2523-2529, 2012.

24. Chua BT, Guo K and Li P: Direct cleavage by the calciumactivated protease calpain can lead to inactivation of caspases. J Biol Chem 275: 5131-5135, 2000. 